\title{
Classification of Viral Hemorrhagic Fever Focusing Ebola and Lassa Fever Using Neural Networks
}

\author{
Nikolai A. K. Steur and Carsten Mueller
}

\begin{abstract}
Despite more than 40 years of experience of dealing with Ebola virus outbreaks and even almost 50 years with Lassa fever virus onsets, these hemorrhagic fevers still remain an omnipresent threat for the human health. One reason for this situation results from the lack of efficient and effective detection equipment for virus recognition and identification. The present paper thematizes the reliable virus cultivation method for classifying pathogens with the aid of electron microscopy and modifies this method by classifying viruses on images through a concepted software component using neural networks.
\end{abstract}

Index Terms-Ebola, image classification, image preprocessing, Lassa fever, neural networks.

\section{INTRODUCTION}

The discovery of Ebola virus and its first records reached to 1976 , in which year two outbreaks of this infectious disease simultaneously occurred in the Central African country Zaire, today's Democratic Republic of Congo, and in South Sudan. In those outbreaks, a total number of 602 infections with Ebola virus were documented and finally 431 human lives were extinguished by this hemorrhagic fever. As a consequence, there can be an alarming mortality rate of approximately 72 percent with respect to these both outbreaks calculated [1].

After this first encounter between humans and Ebola virus, according to the Centers of Disease Control and Prevention $(C D C)$ of the United States, up to the beginning of the year 2014, 27 additional onsets of this infectious illness happened within which overall 1,759 recorded humans were affected and 1,117 of them lost their life. The resulting lethality amounts to the high value of about 63 percent. Despite nearly 40 years of experience of dealing with Ebola outbreaks and the world-wide engagement of many aid organizations, neither the mortality rate could significantly be reduced through technological and medical improvements nor the empirically proven high probability of occurrence for further outbreaks could be decreased. The omnipresent threat to humans caused by virulent Ebola disease was elucidated by the last epidemic from the midyear of 2014 until 2016 when the tremendous number of 28,682 cases of infection and 11,359 deaths were documented [2].

As reasons for the high-risk potential of the Ebola virus for future onsets and epidemics, World Health Organization

Manuscript received May 15, 2018; revised April 30, 2019.

N. A. K. Steur is with the Baden-Wuerttemberg Cooperative State University, BW 74821 Germany (e-mail: nikolai-steur@t-online.de).
(WHO) mentions, expect the diverse ways of virus transmission through human and animal, the loss of a comprehensive surveillance system and the lack of appropriate diagnostical equipment [3], [4]. With respect to the technical equipment, especially it was criticized that no efficient and cheap diagnostic technology is currently available. Because of this long-term omissions on the technical side against Ebola virus, enough data for reliable predictions to forecast further outbreaks could not be collected.

A similar picture was observed in the symptomatically related infectious disease Lassa fever which was discovered in the eponymous Nigerian town Lassa in 1969. Lassa fever, just like Ebola, is one of the hemorrhagic fevers and frequently occurs in the West African countries Nigeria, Sierra Leone, Guinea and Liberia. The primary challenge for fighting against Lassa fever consists of identifying infected living beings because characteristic symptoms are only apparent in 20 percent of all cases, according to fact sheet of the WHO [5]. Furthermore, this very infectious disease spread over body secretions from humans and animals as well whereby in particular rodents play an important role for virus transmission. It is supposed, that every year between 100,000 and 300,000 persons get infected with Lassa fever and approximately 5,000 persons die as result of the symptoms [6]-[8]. Lassa fever especially shows fatal consequences for pregnant women by 29 percent probability of maternal death and 87 percent for losing an unborn child during pregnancy [9]. The current recorded Lassa fever outbreak in Nigeria spells out the risk of this infectious illness for the human health [10]. In the time span from the beginning of 2018 to 4 th March 2018, the international aid organization International Federation of Red Cross and Red Crescent Societies reports about 353 confirmed infections with Lassa fever virus, whereby 110 of these suffers died [11]. The report on the Lassa fever outbreak until 6th May 2018, from Nigeria Centre For Disease Control, makes this situation even more critical by registering an increase of confirmed infections to 423 in two further months [10], [12].

Regarding the consistent fight against Lassa fever, the world-wide organizations face challenges similar to the symptomatic-related Ebola virus. Thus, the lack of a common information system for storing and exchanging clinical pictures across geographical borders can also be cited. In the case of Lassa fever disease, this situation is reinforced through its ambiguous symptoms which can not be explicitly assigned to a specific infectious illness. Besides the in use diagnostic technologies for classification of this disease were criticized for its capacity relating to the resulting costs and the necessary expenditure of time [9], [12]. 


\section{LITERATURE REVIEW}

Beginning with the first contact with life-threatening infectious diseases up to today, like Ebola and Lassa fever, diverse diagnostical methods and techniques for solid identification of virions in organic tissue emerge. At the start of the science, organic material of probands were extracted, cultivated and under high-resolution electron microscopes examined for determining specific virus characteristics [9]. The cultivation of tissue samples and the subsequent identification of the existing pathogens by using electron microscopy, reached a remarkable accuracy wherefore this procedure is often used as first instance classifier at new outbreaks [13]. After a successful classification of the causative virus species, suitable measures can be chosen to contain the recognized illness. Due to the relative great effort caused by laboratory creation and examination of living virus cultures, testing methods like Polymerase Chain Reaction $(P C R)$ were preferred for extensive application and were considered as today's de-facto standard [9], [14]. Virus cultivation as well as PCR method completely solves present dependencies of ambiguous symptomatic during diagnosis. As consequence of the empirical high precision and the relative small effort, PCR is mostly used in daily routine, but electron microscopy is also often used for the first virus species identification and for more exact examinations [9]. As announced earlier, in spite of the high accuracy of the existing diagnostic techniques, there are many aspects which can be criticized. Thus, all known techniques require, more or less, big personal effort and well-trained employees. For this purpose, there are always new innovative and efficient alternatives searched for replacing or complementing existing diagnostic concepts.

The present paper thematizes a modification of the reliable diagnostic method of tissue cultivation, with subsequent pathogen analysis using electron microscopy, by a software component for automatic virus classification. The motivation behind a software-based virus identification within electron microscopical images, is a general improvement of efficiency and effectivity of the classic procedure and the reduction for needed expert knowledge in medicine by analyzing such images.

Further goals of this paper are the realization of the classification software component using the innovative neural networks technology, concepting a potent image preprocessor and parallelizing the corresponding tasks.

As a result, the software component should finally be able to classify two electron microscopical images, despite comparative little resources use.

\section{FOUNDATIONS}

The theoretical foundations consist of three issues which provide information about external virus features within electron microscopical images, neural network basics and a few techniques of image processing.

\section{A. Electron Microscopical Virus Characteristics}

After an infection with a life-threatening virus, a rapid and aimed treatment for protecting patient's life has an essential meaning. To make this possible, an explicit pathogen identification is initially required. As a general rule, based on the patient's symptomatic, hypothesizes for plausible reasons were formulated and then proven by corresponding testing methods. The problem with Ebola, Lassa fever and other hemorrhagic fevers is that these diseases make similar impacts [7]. Furthermore, a special urgency is created by the low incubation time of this virus class about approximately 21 days [1], [7]. As a consequence, if a hemorrhagic fever were suspicious then virus cultivation is a trusted testing method. Hence in the following, the appearance of the viruses Ebola and Lassa fever within electron microscopical images will be discussed. The successive electron microscopical image contains organic material which is contaminated with Ebola virus virions.

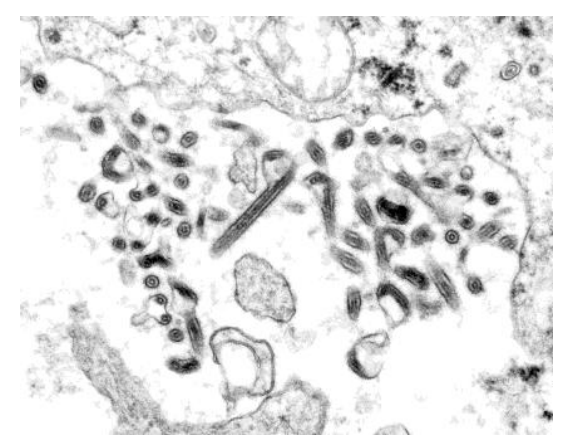

Fig. 1. Electron microscopical image containing Ebola virus virions from the CDC [15].

Fig 1 illustrates an image within Ebola contaminated organic tissue which was created by using electron microscopy. At the image edges there can be seen inconspicuous organic material. The Ebola virus virions are the opaque structures which can be located in the center of the figure. Characteristic for these structures are worm- or tube-like shapes that are common entangled and mostly surrounded by nothing in close environment. It is imaginable that through the entanglement and node forming of virions, they can generate a diversity of shapes whereby classification can get complicated. This situation can especially be assumed for computer vision because on static electron microscopical images, Ebola virus virions be able to generate many diverse pixel patterns.

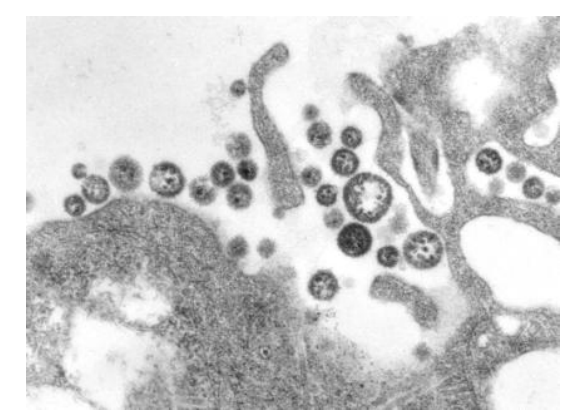

Fig. 2. Electron microscopical image containing Lassa fever virus virions from the CDC [16].

The following Fig. 2 shows Lassa fever virus virions under the electron microscope. The virions are situated as round shapes in the mean height of the picture. All other material can be assigned to the virus classification irrelevant image structures. Noticeable is the fact, that the virions gather in 
empty spaces near vital organic tissue. Thereby the virions form representative accumulations. Another visible feature of Lassa fever virus virions is the almost black inked margin around the circular shape and the also black dots close to the inner border.

In comparison to the related Ebola virus, Lassa fever virions are more uniform shaped, namely as circular structures, which allow an easier general abstraction of this kind of virion. Based on this knowledge, it can be supposed that a software component for virus autodetection should achieve higher hit accuracy during classifications of images with Lassa fever.

\section{B. Neural Networks Basics}

A neural network (NN) at least consists of two layers, namely an input and output layer. The input layer usually consumes a sum of normalized input values which could be interpreted as an external stimulus. For instance, in the case of an intelligent system for image classification, a stimulus can be represented as a vector of pixel values. After the execution of the internal sequential processing steps, the interim results propagate towards the output layer where the final values get calculated. In the last step the final results have to be construed by the application logic. Within every layer of a NN, neurons act as atomic part. The subsequent Fig. 3. illustrates an abstract description of the underlying neuron model. The separated circle in the center of the picture depicts a single artificial neuron. On the left side, three small circles which serve as inputs for the centered neuron can be found. The smallest circle, that contains the lower-case letter $b$, stands for the term bias. The bias input is an additional parameter for a specific artificial neuron and has an independent behavior from actual input vectors by always adding the same value to the resulting input sum [18], [19]. The benefit of using biases is an increase of the resulting parameterization. The other two little circles with the description $H$ with different indices, represent neurons of the direct previous layer which are connected to the neurons of the next layer and operate as part of the input signal.

The upper number shows the index of the corresponding layer and the lower number is the index of the neuron in its layer. The connections between the input value producers and the centered neuron are described by input values that were multiplied with weights $w$ [18].

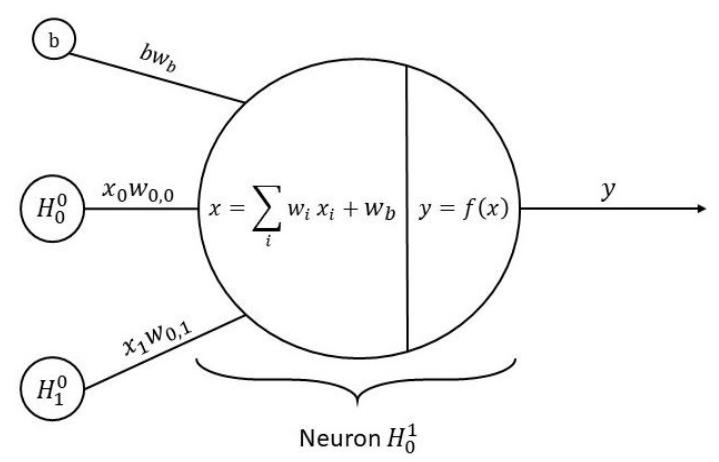

Fig. 3. Basic mathematical model for a neuron, inspired by [17], [18].

In Fig. 3 the input signal for a neuron contains the sum of all weighted inputs and the neuron-specific bias weight. This is possible because every bias usually has the constant value of one and can only be distinguished by its corresponding connection weight [18]. After calculating the input sum, an activation function for computing the neuron output is used. Common utilized activation functions are for instance sigmoid, hyperbolic tangent and hard limiting thresholds [18].

A neural network includes, except the input and output layer, a variable number of hidden layers. Each layer contains an individual number of corresponding neurons. The neurons of the input layer are called input neurons and point out a uniqueness in comparison to the neurons of the other layers. Thus, input neurons have not biases and do not use activation functions. As a consequence, they just reflect the delivered values of the input vector. Besides, the neurons of hidden layers are named hidden neurons and the neurons of the output layer are called output neurons. Through adding further hidden layers with diverse number of hidden neurons to a NN architecture, generally the performance capacity is increasing. At the beginning of the artificial neural networks development, the researcher Rosenblatt mostly examined this technology for uniliteral classifications in the years 1958 and 1962 and named corresponding NNs as Perceptron [20]. Hence, many complex multilayered $\mathrm{NN}$ architectures are described as Multilayer Perceptrons (MLP) until now [18].

The communication between single subsequent layers can be constructed differently. One of the most used architectures are so-called feedforward networks, in which a neuron of a previous layer is fully-connected with neurons of the direct following layer. An equivalent name for MLPs is deep neural networks, because of the resulting deep net architecture [20].

The following Fig. 4 shows an exemplary deep neural network architecture with feedforward propagation. The drawn arrows mark the processing direction. Now, input vectors can forward propagate through each hidden layer up to the output neurons.

There are many methods available for training a NN. The most common learning algorithm is backpropagation which can be understood as the counterpart of forward propagation. Before a learning session can be started, a forward propagation with a training sample is required for computing current output values of the neurons. Afterwards the classification error will be calculated, and the connection weights of each layer will sequentially be adjusted to minimize this error [17], [20].

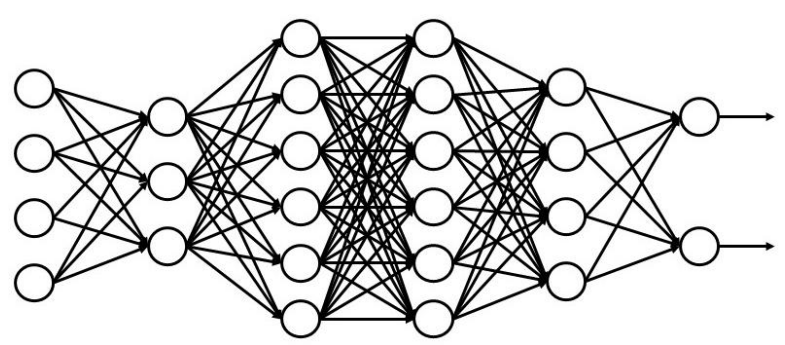

Fig. 4. Fundamental fully-connected neural network architecture.

Today deep neural networks are successful used in many application domains. In the wide spectrum of image recognition using neural networks, there are mostly utilized Convolutional Neural Networks $(C N N)$ s which are characterized by feature extraction and abstraction with the help of various interlayers. As a result of this approach, the 
parameter space extends and allows higher accuracy, but also requires a large number of training samples and sessions [21].

\section{Image Processing}

In image processing the morphologic operations opening and closing are common methods for reinforcing contours and eliminating undesired noise. The behavior of a morphological filter is explicit described by its algorithm and a structure element. The structure element is usually represented as a matrix and consists of the values one and zero. Besides any desired matrix element can be chosen as hot spot which specifies the origin position for applying morphological transformations. An opening filter includes an erosion followed by a dilation. An erosion filter minimizes image objects by using a structure element. Each pixel gets iterative regarded and based on rules modified. An erosion filter compares image regions with the structure element. In binary pictures, if all one-elements of the structure matrix match the regarded image region, the pixel with the hot spot position is set to one, otherwise zero. Dilation in contrast to erosion means the expansion of image objects with the aid of a structure element by comparing the structure element with image regions. After comparing the marked pixel on the hot spot position and its neighborhood with the structure element, the regarded pixel will be manipulated by defined rules. Usually the expansion was concrete caused in binary pictures by comparing the one values of the structure element with the located pixels values on the regarded image. If one pixel of the neighborhood or the hot spot pixel matches with a one-element of the structure matrix, then the hot spot pixel is assigned the number one, otherwise zero [22].

Fig. 5 visualizes the opening filter applying on an image with 25 pixels. At the left upper corner, an input image with a dominating blue object to the left and with background noise to the right can be seen.

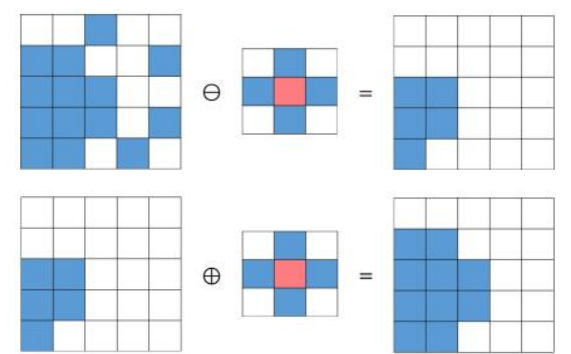

Fig. 5. Exemplary opening filter application on an image with background noise, inspired by [22].

The opening filter is introduced by applying an erosion. The interim result shows that any background noises were removed, but simultaneously the relevant image structure was damaged. For compensating this side effect, a dilation filter is used after this. The dilation expands the minimized object to its original size. As the final result, the background noise was completely eliminated, and relevant structures were retained.

\section{Software ARchitecture}

For the design of a powerful software with NN technology, it is recommended to construct a modular and flexible architecture, because for most application cases, general valid solutions do not exist. Performant NN architectures are often the result of systematic parameter testing with plausible input data. The concepted NN architecture consists of the obligatory input and output layer and a variable number of hidden layers. An additional aspect is that the corresponding number of neurons within each layer should be free to choose. As a consequence of the framework conditions, the output layer has constantly two neurons whereas one stands for Ebola virus recognition rate and the other neuron for the percentage accordance with the Lassa fever virus. The NN uses a fully-connected, multilayered feedforward network. The values for all connection weights should randomly be selected in the initialization phase and correspond to a value between minus and plus one.

The momentum, or also called momentum term, serves as extension for the backpropagation algorithm. Plausible problems of the classical backpropagation algorithm are the oscillation of the error function, the falling into a local minimum or a long training time. These problems can partially be compensated by using the momentum term [18].

The momentum term is described by the equation

$$
w_{j i}(k+1)=w_{j i}(k)+\Delta w_{j i}(k)+\mu \Delta w_{j i}(k-1) .
$$

Reference [18] shows (1). Equation (1) depicts the modification of the classical calculation of the new connection weights after applying the backpropagation algorithm.

The momentum term adds additionally the delta weight of the previous iteration, multiplied by the defined momentum rate. As a result of this approach, rapid gradient changes between two backpropagation iterations will be weakened whereby generally oscillating behavior can be prevented. Another advantage is emerged by the accelerating of gradient descent on routes without significant gradient changes. Thus, the number of training sessions can be remarkable reduced.
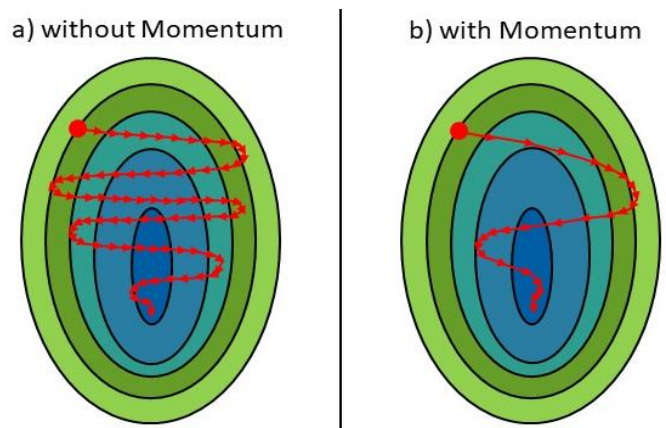

Fig. 6. Visualization of the momentum effect during a learning process inspired by [23], [24].

Fig. 6 schematically visualizes the described advantages. In both subimages, it can be seen a learning curve which is on the right picture influenced by the momentum term and on the left image the classical background algorithm is used. The colored regions represent contour lines, provided that high-altitude regions are light green and the low-altitude regions are dark blue illustrated. The dark blue middle of the drawn ellipse represents the global minimum of the error function. The previously described advantages can be comprehended by the figure. Due to the mentioned benefits 
by using the momentum term within a NN architecture, it is also implemented in the software concept of this paper.

To significantly increase the consequent performance of the classification software and for providing parallelization, it is necessary to concept an additional component for image segmentation.

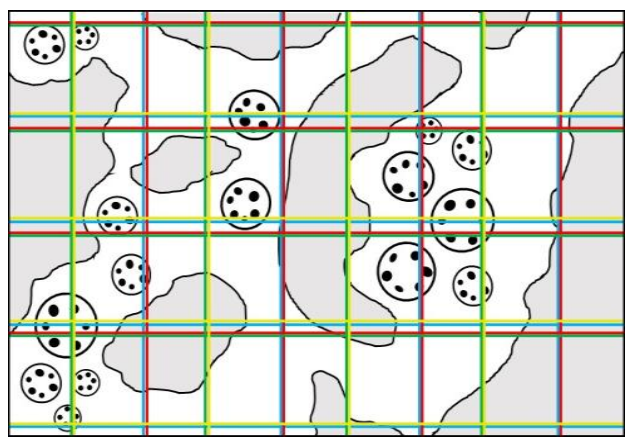

Fig. 7. Multiple segmentation of an input image.

Fig. 7 visualizes possible screenings for multiple segmentation of an input image. The graphic illustrates a schematic electron microscopical image of Lassa fever virus virions which should be classified by the constructed software component. The remaining gray picture objects depict irrelevant organic tissue.

The image segmentation contains a four-phase segmentation which is identifiable through the four different colored grid constructs. The four grids could be distinguished by their colors and various start positions. The blue raster begins at the left upper corner and forms in each grid row four complete rectangles. As a result, on the right side of the input image, it can be seen a left picture section which will be ignored for further processing steps. With a view to the red colored grid, the same image strip emerges to the right because of the screening start position at the left bottom corner. Considering the yellow and green grid, whereas there can be identified an ignored strip on the left side. Additionally, analogous image strips occur at the top and the bottom of the input image, depending on the start position of the segmentation process. This means, if there is an image with odd dimensions referring to width and height, the applied multiple segmentation process profits by cutting out a variety of image segments with different perspectives. Thus, the result of using four segmentation tasks with different start positions, is capturing the entire input image by dividing it in overlapped segments. As a consequence, significant features can be specific searched and one characteristic is extracted and regarded from various perspectives. Besides, unprepossessing cutting up of relevant features is compensated by overlapped multiple segmentation and the chance for a correct virus identification will be increased. For instance, this precondition of odd image dimensions can be created explicitly by appropriate image trimming. The concept of the classification software provides that each excised subimage, represented by one segment, should be classified independently by the developed neural network. A segment can be imagined as an own data structure which contains a subimage, the corresponding classification output vector and the subimage position with respect to the original input image.
Relevant to the NN architecture, the segment dimension should accord the number of input neurons. With the aid of the segments generation, it is possible to classify much smaller images as the original input image. As a result, the applied NN architecture requires a lesser extent for achieving the wanted effect. A simple NN structure enables an increase in speed and an adequate resource efficiency. A further aspect is that the four-phase segmentation process supports concurrency without side effects by storing all segments and using individual classification processes which have a deep copy of the trained neural network in their isolated memory space. Because this parallelism can cause a high memory usage, the simple NN architecture mitigates this problem. After multiple segmentation and concurrent virus classification, the segment results should be collected by a superordinate instance and in the context to the entire input image interpreted.

The preprocessor concept of the classification software takes shape of diverse filter technologies which are combined to a filter chain. The resulting image preprocessor should affect a strong reduction of the NN architecture complexity.

In consequence of this, the throughput speed of image classification will be increased and the early feature extraction should improve classification accuracy.

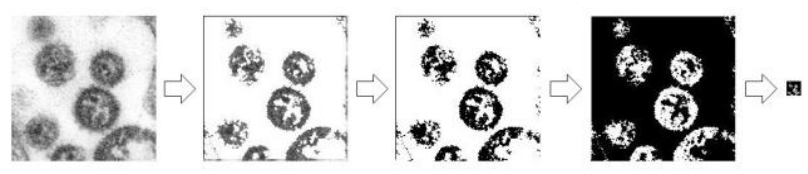

Fig. 8. Filter chain of the image preprocessor.

Fig. 8 illustrates the complete filter chain of the preprocessor with the aid of a snippet from Fig. 2 which represents an image segment with Lassa fever virions. The image segment has the format 200 pixels $\times 200$ pixels. The depicted filter chain uses the named filters opening, precision, inverting and scaling. The opening filter is the first planned element of the filter chain and should provide an emphasis of the characteristic image features. Besides, the opening filter should eliminate irrelevant image structures and background noise. For this purpose, the from the area of digital image processing known technique opening is used and for provisioning a performance increase modified. The modification compromises the definition of an application-specific structure element. The structure element has the form

$$
\left(\begin{array}{lll}
0.0 & 0.8 & 0.0 \\
0.8 & 1.0 & 0.8 \\
0.0 & 0.8 & 0.0
\end{array}\right) .
$$

The used structure element is defined as $3 \times 3$ matrix which resembles a conventional structure element because of its square form with a cross-shaped numerical presentation. The chosen form of presentation does not show that the middle point with a value of one acts as hot spot position. Due to the fact, that each electron microscopical image is presented as grayscale image, the percentage brightness can be described as a value between 0.0 and 1.0 whereby the value 0.0 depicts black and the value 1.0 represents white. If the 4-neighborhood of the actual regarded pixel appreciates many 
light pixels with a brightness starting from 80 percent (equals the value 0.8 in the structure element), then the regarded pixel gets the value 1.0. Regarding electron microscopical images, it may be expected that the relevant image features are depicted by values unequal 1.0. This means that light regions without dark structures contain no information which is needed for classification purposes. Through the application of the opening filter, the background noise will be removed or even completely eliminated. Thus, the regions with less entropy will be reduced. Simultaneous to this process, dark and relevant structures will be emphasized by the opening filter. Fig. 8 demonstrates this method with the first image processing step. The next element within the filter chain is the so-called precision filter.

The precision filter embodies the second element within the filter chain and represents a simple threshold function. This means that this filter compares each grey value of a pixel with a defined threshold. If the concrete regarded pixel value is smaller than the threshold value then the pixel gets the value 1.0, otherwise 0.0 . This rule has to applied to each pixel of the input image. This imaging approach reduces pixel noise as well and highlights significant structures. The inverting filter is the third member of the preprocessor filter chain. This filter serves the inverting of each pixel value. It can be seen that the result image represents the negative of the original picture. The resulting benefit emerges through the nature of the concepted NN architecture because structure pixels get the value 1.0 and empty space pixels are set to 0.0 . Thus, the inverting filter optimally shapes the weighting of the NN input vector. The last filter of the chain is called scaling filter. The task of the scaling filter is the reduction of the image dimensions by specified factors. The scaling operation is realized as affine transformation with the aid of a scaling matrix [25]. The scaling factors for both axes ( $\mathrm{x}$ - and $\mathrm{y}$-axis) are defined as 0.1 . This means that the original image segment with the format of 200 pixels $\times 200$ pixels, is downsized to the dimensions 20 pixels $\times 20$ pixels. Fig. 8 depicts the effect of the scaling filter and finally shows the input image for the NN architecture. The main advantage is that the NN complexity is reduced to only 400 required input neurons. Thus, the virus classification software with an appropriate image preprocessor were fully concepted.

\section{SENSITIVITY ANALYSIS}

In this part of the paper, the parameters activation function, learning rate, momentum rate and number of hidden layers will be considered by applying a sensitivity analysis. For accomplishing a meaningful analysis, a large amount of data must be collected and statistically evaluated. For achieving a high result significance, the number of iterations is set to 10.000 in every scenario and each hidden layer contains 80 neurons. Subsequently, each scenario will be evaluated with four test images of the size 200 pixels $\times 200$ pixels by learning these images within a defined number of training iterations. The scenarios will use identical deep copies of a $\mathrm{NN}$ for providing comparability. One training iteration will contain the backpropagation application for each test image. After one iteration, the average classification rate will be stored. For obtaining optimal sensitivity analysis results, each scenario will for the Ebola and the Lassa fever virus separately be applied regarding the specific output neuron. After collecting the corresponding data for both virus kinds, the data will be analyzed related to the values mean, median and standard deviation.

\section{A. Ebola Virus}

This section presents the calculated metrics for the output value which specifies the recognition rate for the Ebola virus. Scenario00 uses as activation function the sigmoid function and Scenario01 the hyperbolic tangent. The coefficient of both functions has the value 1.0.

For providing a consistent normalization for the final output values, the output neurons within Scenario01 also use the sigmoid function.

TABLE I: DESCRIPTION OF COMPUTED RATIOS BY TESTING ACTIVATION FUNCTION PARAMETER IMPACT

\begin{tabular}{lll}
\hline \hline & Scenario00 & Scenario01 \\
\hline Mean & 0.9753881 & 0.9846675 \\
Median & 0.9867090 & 0.9905209 \\
Standard Deviation & 0.055258224 & 0.028039890 \\
\hline \hline
\end{tabular}

Table I lists all calculated ratios for both scenarios. It can be seen that, within this minimal example, the hyperbolic tangent as activation function produces better results than sigmoid because Scenario01 has higher values of mean and median.

For the statistical analysis of the learning rate parameter, three different scenarios were defined. Scenario02 begins with a learning rate in the amount of 0.001 and the further scenarios in this category have an increase of this learning rate by multiplying it with a factor of ten. Thus, Scenario03 gets a learning rate of 0.01 and Scenario04 has a learning rate of 0.1 .

TABLE II: DESCRIPTION OF COMPUTED RATIOS BY TESTING LEARNING RATE PARAMETER IMPACT

\begin{tabular}{llll}
\hline \hline & Scenario02 & Scenario03 & Scenario04 \\
\hline Mean & 0.8864106 & 0.9753881 & 0.9945368 \\
Median & 0.9370182 & 0.9867090 & 0.9966288 \\
$\begin{array}{l}\text { Standard } \\
\text { Deviation }\end{array}$ & 0.149478620 & 0.055258224 & 0.015157216 \\
\hline \hline
\end{tabular}

Table II illustrates the results of the sensitivity analysis with respect to the learning rate. The tabular overview shows that the mean and median were improved by increasing the value of the learning rate. Surprisingly, the standard deviation of the first and third displayed scenario are similar while the standard deviation of Scenario02 varies widely.

The next three scenarios regard the parameter dependency of the momentum rate in relation to the classification results. This parameter dependency can analogously to the learning rate be examined.

Table III visualizes the statistical results of the sensitivity analysis with variation of the momentum rate. It is apparent that the computed numbers of Scenario05 hardly differ from Scenario06. 
TABLE III: DESCRIPTION OF COMPUTED RATIOS BY TESTING MOMENTUM RATE PARAMETER IMPACT

\begin{tabular}{llll}
\hline \hline & Scenario05 & Scenario06 & Scenario07 \\
\hline Mean & 0.9738323 & 0.9739836 & 0.9753881 \\
Median & 0.9859007 & 0.9859793 & 0.9867090 \\
$\begin{array}{l}\text { Standard } \\
\text { Deviation }\end{array}$ & 0.057718406 & 0.057481422 & 0.055258224 \\
\hline \hline
\end{tabular}

On the contrary, Scenario07 records a comparatively high discrepancy related to the remaining scenarios. Additionally, Scenario07 has a relatively small standard deviation which can be positively interpreted as well. Based on these results, it can be assumed that a systematical variation of the momentum rate allows conclusions about the curve progression of the multidimensional error function, by interpreting error values as gradient changes.

The last three scenarios address the effect of the hidden layer number. One scenario contains one, two or three hidden layers. Within these scenarios, each scenario gets a different randomly generated neural network for applying classifications. Therefore, it can be expected that the initial classification results vary widely from each other. But during the course of the analysis, the results should align to each other.

TABLE IV: DESCRIPTION OF COMPUTED RATIOS BY TESTING NUMBER OF HIDDEN LAYERS PARAMETER IMPACT

\begin{tabular}{llll}
\hline \hline & Scenario08 & Scenario09 & Scenario10 \\
\hline Mean & 0.9091248 & 0.9899566 & 0.9750745 \\
Median & 0.9562285 & 0.9927014 & 0.9835812 \\
$\begin{array}{l}\text { Standard } \\
\text { Deviation }\end{array}$ & 0.139416054 & 0.009501399 & 0.034953616 \\
\hline \hline
\end{tabular}

Table IV shows that Scenario09, which uses for the classification two hidden layers, achieves the values with the highest virus identification precision. Furthermore, it can be discovered that a big discrepancy exists between the means and medians of Scenario08 and Scenario10 because the results of Scenario10 are much more positive.

\section{B. Lassa Fever Virus}

The metrics for the computation of the statistical ratios in this section only refer to the output neuron which is used for signaling percentage accordance with Lassa fever virus virions on electron microscopical images. Regarding the investigation of the solution quality for the recognition of the Lassa fever virus, the parameter dependency concerning the activation function were considered. For this purpose, Scenario00 was again compared to Scenario01 whereby the first scenario uses the sigmoid function and the second one implements the hyperbolic tangent function. The coefficient of both functions is set to 1.0.

The results, which are presented in Table $\mathrm{V}$, reflect the ratios of the corresponding examination. Thus, the utilization of the hyperbolic tangent ensures a proportional high precision. In addition, the standard deviation of Scenario01 is considerably smaller than the standard deviation of Scenario02.

With respect to the equivalent analysis of the Ebola virus, it can be said that the results of the Lassa fever recognition show a higher classification accuracy, whereby this situation depends on different initialization values regarding the output neurons.

TABLE V: DESCRIPTION OF COMPUTED RATIOS BY TESTING ACTIVATION FUNCTION PARAMETER IMPACT

\begin{tabular}{lll}
\hline \hline & Scenario00 & Scenario01 \\
\hline Mean & 0.9802872 & 0.9938611 \\
Median & 0.9875631 & 0.9954158 \\
Standard Deviation & 0.034378117 & 0.005616248 \\
\hline \hline
\end{tabular}

For exploration of the impacts through the choice of the learning rate value, the three regarded scenarios implement the values $0.001,0.01$ and 0.1 in the presented order.

TABLE VI: DESCRIPTION OF COMPUTED RATIOS BY TESTING LEARNING RATE PARAMETER IMPACT

\begin{tabular}{llll}
\hline \hline & Scenario02 & Scenario03 & Scenario04 \\
\hline Mean & 0.9227124 & 0.9802872 & 0.9950180 \\
Median & 0.9521416 & 0.9875631 & 0.9964724 \\
$\begin{array}{l}\text { Standard } \\
\text { Deviation }\end{array}$ & 0.095713905 & 0.034378117 & 0.005994450 \\
\hline \hline
\end{tabular}

Table VI contains the results of the statistical computations for obtaining the ratios of the scenarios Scenario02, Scenario03 and Scenario04. It can be seen that with the increase of the learning rate, the classification quality grows simultaneously. It is important to note that the results have to interpreted in the context of the used minimal example.

The scenarios for analyzing parameter dependencies of the momentum term are identical constructed to the scenarios of the Ebola virus sensitivity analysis.

TABLE VII: DESCRIPTION OF COMPUTED RATIOS BY TESTING MOMENTUM RATE PARAMETER IMPACT

\begin{tabular}{llll}
\hline \hline & Scenario05 & Scenario06 & Scenario07 \\
\hline Mean & 0.9791679 & 0.9792763 & 0.9802872 \\
Median & 0.9868863 & 0.9869518 & 0.9875631 \\
$\begin{array}{l}\text { Standard } \\
\text { Deviation }\end{array}$ & 0.035976007 & 0.035822371 & 0.034378117 \\
\hline \hline
\end{tabular}

TABLE VIII: DESCRIPTION OF COMPUTED RATIOS BY TESTING NUMBER OF HIDDEN LAYERS PARAMETER IMPACT

\begin{tabular}{llll}
\hline \hline & Scenario08 & Scenario09 & Scenario10 \\
\hline Mean & 0.9325247 & 0.9852404 & 0.9823994 \\
Median & 0.9592426 & 0.9893522 & 0.9880108 \\
$\begin{array}{l}\text { Standard } \\
\text { Deviation }\end{array}$ & 0.073794106 & 0.014162786 & 0.020972776 \\
\hline \hline
\end{tabular}

Table VII shows that the calculated ratios from Scenario05 and Scenario06 are hardly different. In contrast, the results of Scenario07 vary widely from the other results and achieve the highest solution quality.

The effect of the chosen number of hidden layers in the implemented neural network architecture is regarded. Besides, the scenarios describe the use of one, two or three hidden 
layers. By applying the sensitivity analysis, each scenario gets a new neural network with random initialization values.

Table VIII presents the investigated ratios to the corresponding scenarios. Scenario09 reaches the best results whereby Scenario10 exhibits similar results.

On the contrary, the ratios of Scenario08 deviate significantly in a negative way.

\section{PROOF OF CONCEPT}

As mentioned in the introduction, the defined proof of concept (PoC) demands one correct image classification for each virus species.

\section{A. Neural Network Architecture}

The chosen NN architecture, for the successful handling of the defined PoC, is importantly influenced by the constructed image preprocessor. Thus, the preprocessor predetermines because of its internal scaling filter the number of input neurons. Additionally, the filter chain of the preprocessor relieves the implemented $\mathrm{NN}$ by providing prepared image segments. This leads to an immediate reduction of needed NN architecture complexity and hence to a high resource efficiency regarding to memory usage and computing effort. The realized NN architecture consists of five layers. In consequence of the preprocessor output as compressed image with the format 20 pixels $\times 20$ pixels, the input layer includes 400 neurons. The output layer contains two neurons whereby one signals the percentage recognition of Ebola virus and the other one analogously indicates the percentage accordance with Lassa fever. The numbers of neurons within the input and output layer are framework conditions determined by the image preprocessor and the underlying classification problem. The rest of the architecture was proved based on systematical testing. As activation function for all neurons in the neural network is the sigmoid function used because of its general wide distribution and application in the area of image classification with neural networks. The inside of the NN comprises three hidden layers which contain in the named order, starting with the layer to the left of the input layer up to the direction to the output layer, 30, 50 and 30 neurons. Due to the reduction and subsequent expansion of the neuron numbers within the hidden layers, image features were firstly summarized and after that expanded. The described architecture allows the resulting NN to solve the existing virus classification problem with a high accuracy.

\section{B. Training Setup}

The training for achieving the PoC has 500 iterations. Within one iteration, the backpropagation algorithm is applied to the whole training data set. The training data set contains 98 images and therefore in total it was required 49.000 iterations for the complete learning process. The training data set composes of 26 Lassa fever virus electron microscopical images, 35 Ebola virus images and 35 negative examples with no virions on it. One training data image equals a subimage with the dimensions 200 pixels $\times 200$ pixels. This approach is necessary because there are not many electron microscopical images of Lassa fever or Ebola virus available for public access. Hence, the existing electron microscopical images were segmented to 200 pixels $\times 200$ pixels subimages. During training, the learning rate obtain the value of 0.05 and the momentum rate is the value 0.1 assigned.

\section{Evidence of Performance}

After the completion of one classification, the results will be visualized in two ways in the implemented frontend. One way is the graphical localization in the entire input image and the other one is an overall assessment with corresponding outcome interpretation. The detection of Ebola virus produces red rectangles and the recognition of Lassa fever virus is marked through yellow rectangles.

The following Fig. 9 illustrates a screenshot which depicts the frontend of the implemented software after a classification process. The concepted and realized software project was named as Artificial Intelligence for Microscopical Image autodEtection (AIMIE).

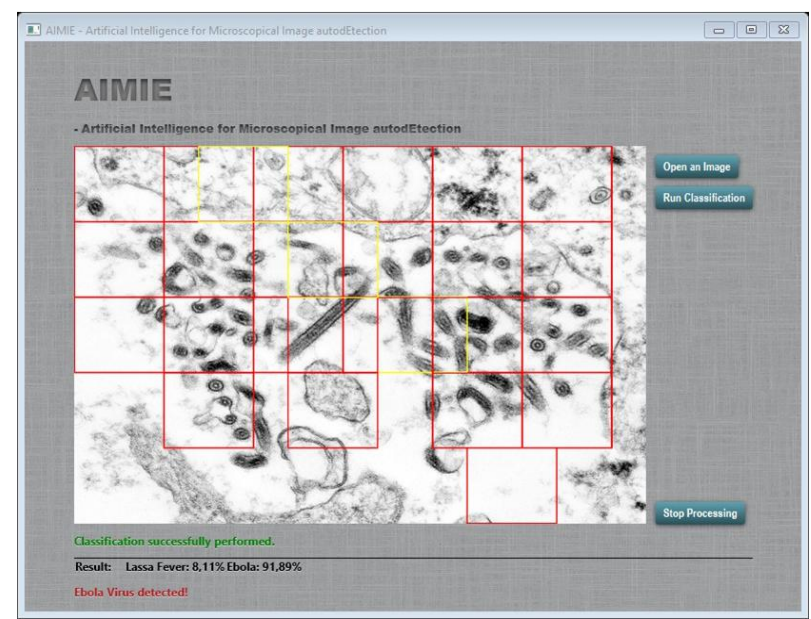

Fig. 9. Results of an automatic classification for an electron microscopical input image with Ebola virions.

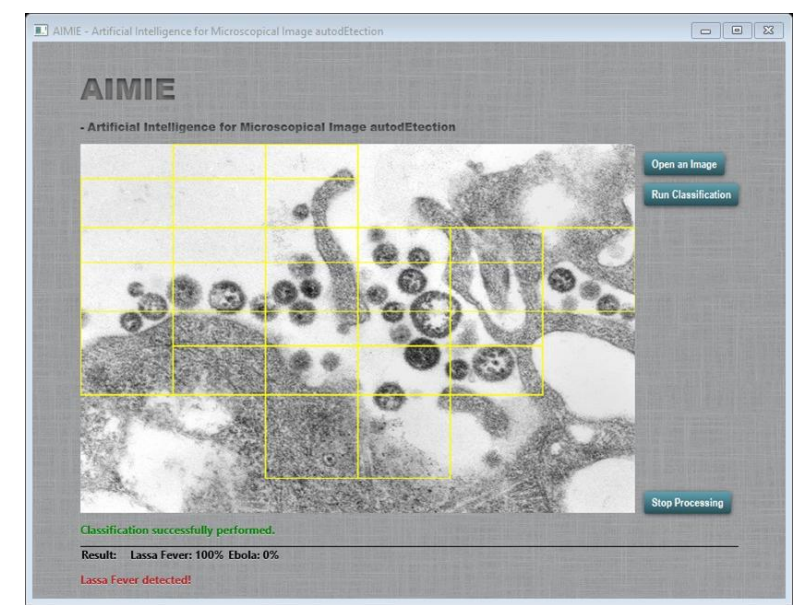

Fig. 10. Results of an automatic classification for an electron microscopical input image with Lassa fever virions.

As input image serves an electron microscopical image of organic tissue which is contaminated with Ebola virus virions [15]. It can be seen that there is a high recognition rate of Ebola virus virions within the input image and only occasional Lassa fever virions were detected. Beneath the displayed electron microscopical image, is visualized an information text, result details and a corresponding textual interpretation. The percentage result for Ebola is calculated by the number of the identified red segments divided by the 
number of yellow rectangles. The Lassa fever percentage value is analogously computed.

The final results show an Ebola virus accordance of approximately 92 percent and a Lassa fever recognition rate of about 8 percent. Thus, the Ebola virus was successfully identified and the first part of the defined PoC is achieved.

The subsequent screenshot represents the software frontend after a classification process with an electron microscopical input image which contains one, with Lassa fever virus infected, tissue specimen [16].

After the application of the classification process the best possible result is reached. The result details depict a Lassa fever detection with 100 percent probability. Fig. 10 also shows that not just the trained data were successfully classified but through the multiple and parallelized segmentation many further virions were successfully localized. The way of visualization is in this example advantageously as well because the relevant image characteristics were complete marked by the software. In consequence of this, the mentioned PoC was achieved by correctly classifying the Ebola and Lassa fever viruses on electron microscopical images.

\section{CONCLUSION}

The underlying paper showed an alternative method for classifying hemorrhagic fever pathogens by automatically analyzing electron microscopical images of organic tissue. The classification was realized with the innovative technology of neural networks and tested with images of the viruses Ebola and Lassa fever. Additionally, the neural network technology was combined with a powerful image preprocessor which consists of diverse filter operations. The expected performance of the classification software was proven by the correct identification of virions on electron microscopical images of Ebola and Lassa fever. The resulting classification software could affect a significant reduction of personal efforts related to the manual examination and identification of viruses on electron microscopical images. This means that the classification software could be used for automatic preinvestigations or recall studies of virus cultivations. As a consequence of this, human intervention is only required for verifying the classification results.

Furthermore, the software highlights the image segments with high probability of contaminated tissue on it, whereby trained personal should be able to accelerate their virus identification processes. The last aspect is that the software component, in combination with a comprehensive information system for collecting electron microscopical virus images, could increase classification accuracy as compared to expert examinations. All in all, it can be said that the underlying problem of this paper was solved by presenting a new measure for the effective and efficient fight against future virus outbreaks. Of course, it still remains necessary to continuously search for further measures for fighting against life-threatening diseases and to combine innovative approaches with the current state of technology.

\section{ACKNOWLEDGMENT}

"I respect and thank Dr. Carsten Mueller, for providing me an opportunity to do this research project and giving me all support and guidance to complete this with highest quality. I am very thankful to him for providing such an excellent support and guidance."

\section{REFERENCES}

[1] B. P. Bell, I. K. Damon, D. B. Jernigan, T. A. Kenyon, S. T. Nichol et al., "Overview, control strategies, and lessons learned in the cdc response to the 2014-2016 Ebola epidemic," Morbidity and Mortality Weekly Report (MMWR), vol. 65, no. 3, pp. 4-11, July 2016.

[2] Centers for Disease Control and Prevention. (July 28, 2017). Outbreaks Chronology: Ebola Virus Disease. [Online]. Available: https://www.cdc.gov/vhf/ebola/outbreaks/history/chronology.html

[3] Geneva: World Health Organization, World Health Statistics 2017. Monitoring Health for SDGs, Sustainable Development Goals, p. 46, 2017.

[4] WHO Ebola Response Team, "After Ebola in West Africa Unpredictable risks, preventable epidemics," The New England Journal of Medicine, pp. 594-595, August 2016.

[5] World Health Organization. Fact sheet: Lassa fever. (July 2017). [Online].

Available: http://www.who.int/mediacentre/factsheets/fs179/en/

[6] A. R. Mubo, "Lassa fever: A threat to nigeria economy a paper presented at," IOSR Journal of Nursing and Health Science, vol. 7, issue 1 ver. IV, p. 10, January/February 2018.

[7] V. Raabe and J. Koehler, "Laboratory diagnosis of lassa fever," Journal of Clinical Microbiology, vol. 55, issue 6, p. 1629, June 2017.

[8] World Health Organization. (2018). Presentation: Introduction to Lassa fever - Managing infectious hazards. [Online]. Available: http://origin.who.int/emergencies/diseases/lassa-fever/lassa-fever-pres entation.pdf

[9] J. O. Olayiwola and A. S. Bakarey, "Epidemiological trends of lassa fever outbreaks and insights for future control in Nigeria," International Journal of Tropical Disease \& Health, vol. 24, issue 4, pp. 6-9, July 2017.

[10] Nigeria Centre For Disease Control, "2018 Lassa fever outbreak in Nigeria," Situation Report - Nigeria Centre For Disease Control (NCDC), May 6, 2018.

[11] International Federation of Red Cross and Red Crescent Societies, "Emergency Plan of Action (EPoA) - Nigeria: Lassa acute viral haemorrhagic fever," International Federation of Red Cross and Red Crescent Societies (IFRC), p. 1, March 2018

[12] D. J. Omeh, G. I. Achinge, and P. O. Echekwube, "Lassa fever in West Africa: A clinical and epidemiological review," Journal of Advances in Medicine and Medical Research, vol. 24, issue 6, pp. 1-9, November 2017.

[13] C. S. Goldsmith, T. G. Ksiazek et al., "Cell culture and electron microscopy for identifying viruses in diseases of unknown cause," Emerging Infectious Diseases, vol. 19, no. 6, pp. 886-890, June 2013.

[14] S. K. Gire, A. Goba, K. G. Andersen et al., "Genomic surveillance elucidates Ebola virus origin and transmission during the 2014 outbreak," Science, vol. 345, issue 6202, pp. 1369-1372, September 2014.

[15] Centers for Disease Control and Prevention/C. Goldsmith. Created by centers for disease control and prevention (CDC) microbiologist cynthia Goldsmith, this transmission electron microscopic (TEM) image revealed some of the ultrastructural morphology displayed by numerous Ebola virus virions in this specimen. [Online]. Available: https://phil.cdc.gov/details_linked.aspx?pid=1834

[16] C. Goldsmith, P. Rollin and M. Bowen. This transmission electron microscopic (TEM) image depicted numbers of Lassa virus virions adjacent to some cell debris. Centers for Disease Control and Prevention. [Online]. Available: https://phil.cdc.gov/Details.aspx?pid=8700

[17] W. Ertel, Computational Intelligence: Grundkurs Künstliche Intelligenz - Eine praxisorientierte Einführung, Wiesbaden: Springer Vieweg, pp. 265-294, 2016.

[18] F. M. Soares and A. M. F. Souza, Neural Network Programming with Java - Create and Unleash the Power of Neural Networks by Implementing Professional Java Code, Birmingham: Packt Publishing, 2017, pp. 1-6, 60-68, 80 .

[19] M. A. Nielson, Neural Networks and Deep Learning, Determination Press, 2015, ch. 1.

[20] I. Goodfellow, Y. Bengio, and A. Courville, Deep Learning, Cambridge, MA: MIT Press, 2017, pp. 14, 163-166, 197, 494-498. 
[21] M. Oquab, L. Bottou, I. Laptev, and J. Sivic, "Learning and transferring mid-level image representations using convolutional neural networks," in Proc. 2014 IEEE Conference on Computer Vision and Pattern Recognition (CVPR), pp. 1-4.

[22] W. Burger and M. J. Burge, Digitale Bildverarbeitung - Eine algorithmische Einführung mit Java, Wiesbaden: Springer Vieweg, 2015, $3^{\text {rd }}$ ed. pp. 191-197, 203-205.

[23] G. J. B. Orr. (April 2018). Momentum term figure. [Online]. Available: https://www.willamette.edu/ gorr/classes/cs449/figs/valley1.gif

[24] G. J. B. Orr. (April 2018). Momentum term figure. [Online]. Available: https://www.willamette.edu/ gorr/classes/cs449/figs/valley2.gif

[25] P. Hartmann, Mathematik für Informatiker - Ein Praxisbezogenes Lehrbuch, Wiesbaden: Springer Vieweg, 2015, pp. 252-254.

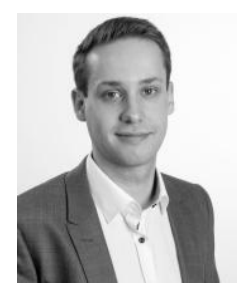

Nikolai A. K. Steur studies applied informatics in the Baden-Wuerttemberg Cooperative State University at Germany.

As part of his dual study, he works as a software developer for an information systems company in Baden-Wuerttemberg in Germany which acts as software supplier for its corresponding retail concern. 